\title{
Novel Method for 5G Systems NLOS Channels Parameter Estimation
}

\author{
Vladeta Milenkovic, ${ }^{1}$ Stefan Panic, ${ }^{2}$ Dragan Denic, ${ }^{1}$ and Dragan Radenkovic ${ }^{1}$ \\ ${ }^{1}$ Faculty of Electrical Engineering, University of Niš, Niš, Serbia \\ ${ }^{2}$ Faculty of Natural Science and Mathematics, University of Pristina, Pristina, Serbia
}

Correspondence should be addressed to Stefan Panic; stefanpnc@yahoo.com

Received 12 November 2016; Revised 4 February 2017; Accepted 12 April 2017; Published 11 May 2017

Academic Editor: Larbi Talbi

Copyright ( 2017 Vladeta Milenkovic et al. This is an open access article distributed under the Creative Commons Attribution License, which permits unrestricted use, distribution, and reproduction in any medium, provided the original work is properly cited.

For the development of new 5G systems to operate in mm bands, there is a need for accurate radio propagation modelling at these bands. In this paper novel approach for NLOS channels parameter estimation will be presented. Estimation will be performed based on LCR performance measure, which will enable us to estimate propagation parameters in real time and to avoid weaknesses of ML and moment method estimation approaches.

\section{Introduction}

Exploitation of unused $\mathrm{mm}$ wave spectrum (spectrum between 6 and $300 \mathrm{GHz}$ ) is an efficient solution for meeting the standards for $5 \mathrm{G}$ networks enormous data demand growth explosion. Because of that characterization and modelling of such channel propagation in urban environments is one of most important tasks in developing novel 5G mobile access networks. Many propagation studies, performed at these bands, for these types of applications, consider lineof-sight (LOS) scenarios [1-3]. However, non-line-of-sight (NLOS) scenarios occur more often. They not only occur in cases when transmitting and receiving antenna are separated by obstructions and there is no clear optical path between the antennas, but also occur in cases when there is indeed clear optical path between the antennas, but antennas are not aligned or boresight [4]. Various outdoor propagation measurements conducted in urban environments have shown that, for reasonable level of signal-to-noise ratio (SNR), that is, higher than $5 \mathrm{~dB}$, NLOS signals have stable first arriving signal levels, even if they are weaker than stronger, laterarriving multipath components, counter to LOS signals with strongest first arriving component at the reception. Measurements provided at $38 \mathrm{GHz}$ (base station-to-mobile access scenario [5] and Peer-to-Peer Scenario [6]), $60 \mathrm{GHz}$ (Peerto-Peer Scenario and vehicular scenario [7]), and $73 \mathrm{GHz}$ [8] have clearly identified existence of NLOS conditions.

One of the most intensively used statistical models for characterizing the complex behavior and random nature of NLOS fading envelope is the Nakagami- $m$ distribution. Nakagami- $m$ fading model closely approximates data values that are obtained by providing real-time measurements in indoor and outdoor wireless environments and is often used in analyzing propagation performances in $5 \mathrm{G}$ systems [914]. In [15-17] for the purpose of modelling observed $5 \mathrm{G}$ system propagation properties the Nakagami $m$ parameter is directly computed from the measured data. As a general fading distribution, Nakagami- $m$ fading model includes in itself other distributions and its simple family form allows obtaining closed-form analytical results for wireless communication link standard performance criterion measures. Namely, Nakagami- $m$ fading model can also be transformed into Ricean fading model, by expressing $m$ parameter in the function of Ricean $K$ factor, as $m=(1+K)^{2} /(1+2 K)$ [18]. In that manner characterizing of the behavior of LOS fading envelope could be also performed. The Nakagami$m$ fading exploits Nakagami probability density function (PDF) for the random envelope of received signal which is 
written in the function of two parameters: scale parameter and shape parameter, called the fading severity parameter or $m$-parameter. Determining $m$ is a problem in Nakagami PDF estimation. For observed set of empirical fading signal data, the value of distribution parameter $m$ should be estimated from it, in order to use the Nakagami- $m$ distribution to model a given set of values. Acquaintance of the $m$ parameter is mandatory for the optimal reception of signals in Nakagami$m$ fading environment [19], and in such case parameter $m$ should be determined very accurately and very fast. Acquaintance of the $m$ parameter is also necessary in the transmitter adaptation process, where its value could be feedback with respect to the channel information. Two most wellknown procedures used for the estimation of the Nakagami$m$ fading parameter, $m$, are (1) maximum likelihood (ML) estimation and (2) moment-based estimation. ML-based $m$ parameter estimation problem reduces to the problem of solving some transcendental equations written in the form of logarithmic and digamma functions. During the process of Gamma shape parameter estimation authors of [20] have developed most famous ML-based estimator of $m$ parameter. Recently, another two approximate ML-based estimators have been proposed in [21], where estimation is carried out by observing approximations of digamma function, that is, first-order approximation and second-order approximation. Opposite approach to the ML estimation of $m$ parameter is the moment-based estimation. Observing the second and the fourth Nakagami- $m$ sample moments, estimation of parameter $m$ was carried out in [22]. An improvement of proposed method can be found in [23] where parameter $m$ estimation is carried on capitalizing on the first and the third sample moments. Group of new moment estimators form based on noninteger sample moments (estimators based on integer sample moment are their special cases), along with simulation study, was proposed in [24]. The generalized method of moments (GMM) is introduced for the estimation of the Nakagami-m fading parameter in [25]. However, it is known that sample moments are often subjected to the effects of outliers (even a small portion of extreme values, outliers, can affect the Gaussian parameters, especially the higher order moments). Moreover, occurrence of outliers is especially problematic when higher order sample moments are used for estimation, since estimation inaccuracy arises in such cases. Providing the best moment-based estimator is still major issue that should be addressed. Specifically, the level crossing rate (LCR), which provides us with a measure of the average number of crossings per second at which the envelope crosses a specified signal level in positive or negative direction, is an important second-order statistical quantity that characterizes the rate of occurrence of fade [18]. However, analytical solution for the LCR often depends mainly on the envelope distribution of the considered process.

\section{Estimation Methods}

2.1. Estimation for Known Power Parameter $\Omega$. Various power estimation techniques have been implemented over years with some advantages and disadvantages. Accurate estimation of the average power of received fading signal is crucial for many reasons. Namely, power control and handoff decisions in wireless communications are mainly based on the accurate estimation of the average signal power. Wireless communication link quality is also indicated through some system criterion measures, such as channel access, hand-off, and power control, that can be determined mainly based on the local mean signal levels. For example, the fading signal power could be estimated by using (38) from [26] without requiring any other parameter to be estimated.

Let $z$ denote the random envelope of received Nakagami$m$ faded signal, and let $\dot{z}$ denote first derivative of $z$ with respect to time. The joined probability density function (JPDF) of $z$ and $\dot{z}$ is then denoted with $p_{z \dot{z}}(z \dot{z})$. The level crossing rate (LCR) at the envelope $z$ is defined as the rate at which a fading signal envelope crosses level $z$ in a positive or a negative direction and is analytically expressed by formula [18]:

$$
N_{z}(z)=\int_{0}^{\infty} \dot{z} p_{\dot{z} z}(\dot{z}, z) d \dot{z}
$$

JPDF can be expressed through the PDF of Nakagami$m$ faded signal envelope, $p_{z}(z)$, and conditioned PDF of envelope time derivative, $p_{\dot{z} \mid z}(\dot{z} \mid z)$, as $p_{\dot{z} z}(\dot{z}, z)=p_{\dot{z} \mid z}(\dot{z} \mid$ $z) \times p_{z}(z)$. PDF expression for Nakagami- $m$ faded signal envelope, written in terms of two fading parameters, $m$ and $\Omega$, is given with

$$
p_{z}(z)=\frac{2 m^{m} z^{2 m-1}}{\Omega^{m} \Gamma(m)} \exp \left(-m \frac{z^{2}}{\Omega}\right),
$$

where $\Gamma(a)$ denotes the Gamma function [27]. Common explanation of parameters $m$ and $\Omega$ is that $\Omega$ defines average power of faded signal; that is, $\Omega=E\left(z^{2}\right)$, while parameter $m$ describes severity of fading process. As seen from [18] envelope time derivative, $p_{\dot{z} \mid z}(\dot{z} \mid z)$, is zero-mean Gaussian random process, defined with

$$
p_{\dot{z} \mid z}(\dot{z} \mid z)=\frac{1}{\sqrt{2 \pi} \sigma_{\dot{z}}} \exp \left(-\frac{\dot{z}^{2}}{2 \sigma_{\dot{z}}^{2}}\right)
$$

and variance of $\sigma_{\dot{z}}^{2}=\pi^{2} f_{m}^{2} \Omega / m$. Here $f_{m}$ is denoted by maximal Doppler shift frequency.

After substituting (2) and (3) into (1), LCR expression can be presented as

$$
N_{z}(z)=\frac{m^{m-1 / 2} \sqrt{2 \pi} f_{m}}{\Omega^{m-1 / 2} \Gamma(m)} z^{2 m-1} \exp \left(-m \frac{z^{2}}{\Omega}\right) .
$$

In order to determine value of parameter $m$ as a function of received signal level and the number of times at which level is crossed, for known value of $\Omega$, we only need to observe two signal levels, that is, $z_{1}$ and $z_{2}$ and rates at which Nakagami$m$ faded signal envelope has crossed those two levels, that is, $N_{z_{1}}$ and $N_{z_{2}}$ that can be determined as

$$
\begin{aligned}
& N_{z_{1}}=\frac{m^{m-1 / 2} \sqrt{2 \pi} f_{m}}{\Omega^{m-1 / 2} \Gamma(m)} z_{1}^{2 m-1} \exp \left(-m \frac{z_{1}^{2}}{\Omega}\right), \\
& N_{z_{2}}=\frac{m^{m-1 / 2} \sqrt{2 \pi} f_{m}}{\Omega^{m-1 / 2} \Gamma(m)} z_{2}^{2 m-1} \exp \left(-m \frac{z_{2}^{2}}{\Omega}\right) .
\end{aligned}
$$


TABLE 1: Comparison between parameter $m$ value obtained by Matlab simulation over $N=100000$ samples of Nakagami- $m$ vector $z$ and value of $m$ parameter estimated by using (6) from this letter $\left(z_{1}=\operatorname{mean}(z), z_{2}=\operatorname{mean}(z)-\operatorname{var}(z)\right.$, and $\left.\Omega=E\left(z^{2}\right)\right)$.

\begin{tabular}{lccccccccc}
\hline$m$ & 1 & 1.25 & 1.5 & 1.75 & 2 & 2.25 & 2.5 & 2.75 & 3 \\
\hline$m_{\text {estimated }}$ & 1.071 & 1.2403 & 1.506 & 1.7507 & 2.009 & 2.2509 & 2.518 & 2.7712 & 3.0121 \\
\hline
\end{tabular}

TABLE 2: Comparison between parameter $m$ value obtained by Matlab simulation over $N=1000000$ samples of Nakagami- $m$ vector $z$ and value of $m$ parameter estimated by using (6) from this letter $\left(z_{1}=\operatorname{mean}(z), z_{2}=\operatorname{mean}(z)-\operatorname{var}(z)\right.$, and $\left.\Omega=E\left(z^{2}\right)\right)$.

\begin{tabular}{lccccccccc}
\hline$m$ & 1 & 1.25 & 1.5 & 1.75 & 2 & 2.25 & 2.5 & 2.75 & 3 \\
\hline$m_{\text {estimated }}$ & 1.038 & 1.2462 & 1.5042 & 1.7503 & 2.003 & 2.2509 & 2.512 & 2.7683 & 3.0117 \\
\hline
\end{tabular}

Now, from (5) parameter $m$ could be expressed in terms of $z_{1}$, $z_{2}, N_{z_{1}}$, and $N_{z_{2}}$ as

$$
m=\frac{\ln \left(N_{z_{1}} / N_{z_{2}}\right)+\ln \left(z_{1} / z_{2}\right)}{2 \ln \left(z_{1} / z_{2}\right)+\left(z_{2}^{2}-z_{1}^{2}\right) / \Omega}
$$

2.2. Real Time Parameter $m$ Estimation for Unknown $\Omega$. In order to determine value of parameter $m$ as a function of received signal level and the number of times at which level is crossed, for known value of $\Omega$, we now need to observe three signal levels, that is, $z_{1}, z_{2}$, and $z_{3}$ and rates at which Nakagami- $m$ faded signal envelope has crossed those three levels, that is, $N_{z_{1}}, N_{z_{2}}$, and $N_{z_{3}}$. For envelope level $z_{3}$, LCR $N_{z_{3}}$ can be expressed as follows:

$$
N_{z_{3}}=\frac{m^{m-1 / 2} \sqrt{2 \pi} f_{m}}{\Omega^{m-1 / 2} \Gamma(m)} z_{3}^{2 m-1} \exp \left(-m \frac{z_{3}^{2}}{\Omega}\right) .
$$

Now, in similar manner parameter $m$ could be expressed in terms of $z_{1}, z_{2}, z_{3}, N_{z_{1}}, N_{z_{2}}$, and $N_{z_{3}}$ as

$$
\begin{aligned}
m= & \frac{1}{2}(1 \\
& \left.+\frac{\left(z_{2}^{2}-z_{1}^{2}\right) \ln \left(N_{z_{1}} / N_{z_{3}}\right)-\left(z_{3}^{2}-z_{1}^{2}\right) \ln \left(N_{z_{1}} / N_{z_{2}}\right)}{\left(z_{2}^{2}-z_{1}^{2}\right) \ln \left(z_{1} / z_{3}\right)-\left(z_{3}^{2}-z_{1}^{2}\right) \ln \left(z_{1} / z_{2}\right)}\right)
\end{aligned}
$$

while now

$$
\Omega=\frac{m\left(z_{1}^{2}-z_{2}^{2}\right)}{\ln \left(N_{z_{2}} / N_{z_{1}}\right)+(2 m-1) \ln \left(z_{1} / z_{2}\right)} .
$$

\section{Numerical Results}

In Table 1 comparison between parameter $m$ value obtained by Matlab simulation over $N=100000$ samples of Nakagami$m$ vector $z$ and value of $m$ parameter estimated by using (6) from this paper has been shown. Nakagami- $m$ random envelope vector $z$ was obtained from Gaussian random processes by using its property that $z=\sqrt{\sum_{i=1}^{m}\left(X_{i}^{2}+Y_{i}^{2}\right)}$, where $X_{i}$ and $Y_{i}$ are zero-mean normally distributed Gaussian random processes, $N\left(0, \sigma^{2}\right)$ [18]. LCR levels $z_{1}$ and $z_{2}$ can be selected

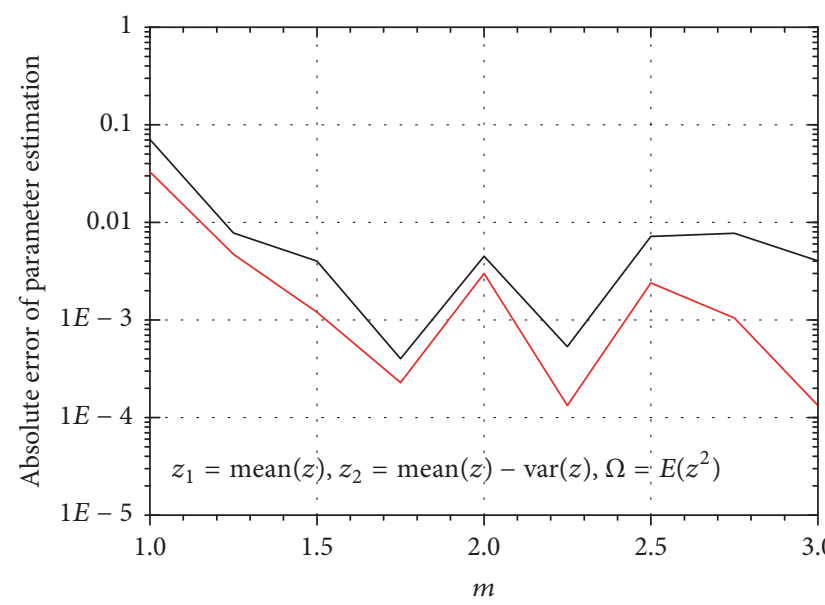

$-N=100000$

$-N=1000000$

FIGURE 1: Absolute error of parameter estimation.

in a way that $z_{1}$ is level with highest number of crosses, while level $z_{2}$ is selected in the near environment of $z_{1}$. Since it is known that envelope level $z_{1}$ for which $N_{z_{1}}$ has maximal value is probably the one for each stand $z_{1}=\operatorname{mean}(z)$, level $z_{1}$ can be selected in that way, while $z_{2}$ level can be selected in the manner that $z_{2}=\operatorname{mean}(z)-\operatorname{var}(z)$. We will observe $m$ parameter values in the range of $m \in[1,3]$, since in [15] it has been shown that values from this range of $m$ parameter correspond to the case of measurements performed at $60 \mathrm{GHz}$ over NLOS channel conditions, when the user was mobile in a range of different indoor and outdoor small cell scenarios. After generating Nakagami- $m$ fading processes, characterized with $m \in[1,3]$, and defining LCR levels for each as explained, by using proposed method based on number of level crosses, we have obtained $m_{\text {estimated }}$ for each of the generated process. As it can be seen from Table 1, shown results provide very good match and proposed method has good accuracy. In Table 2 comparison between parameter $m$ value obtained by Matlab simulation over $N=$ 1000000 samples of Nakagami- $m$ vector $z$ and value of $m$ parameter estimated using (6) from this paper has been shown. Absolute error obtained for $m$ parameter estimation for both cases has been presented in Figure 1 .

By using proposed method Nakagami $m$ parameter can be efficiently determined from various measurement, provided 
for 5G channel characterization, as ones in [15-17], instead of parameter $m$ computation by using moment-based model or the ML estimation. By using proposed method when expressing $m$ parameter in the function of Ricean $K$ factor, as $m=(1+K)^{2} /(1+2 K)$, characterization of the behavior of LOS fading scenarios could be also performed.

\section{Conclusion}

Novel approach for Nakagami $m$ parameter estimation based on LCR has been proposed in this paper. It has been shown that the proposed method not only avoids weaknesses of ML and moment method estimation approaches, but also is quite accurate, simple for realization, and can be used in real time. This interesting approach could also be used for fading parameters estimation of various NLOS channels in real time by using channel emulators for short, subwavelength distances in $5 \mathrm{G}$ multielement antenna realizations.

\section{Conflicts of Interest}

The authors declare that they have no conflicts of interest.

\section{Acknowledgments}

This work has been supported by Project III44406.

\section{References}

[1] M. Kyro, V. Kolmonen, and P. Vainikainen, "Experimental propagation channel characterization of $\mathrm{mm}$-wave radio links in urban scenarios," IEEE Antennas and Wireless Propagation Letters, vol. 11, pp. 865-868, 2012.

[2] J. C. Liberti and T. S. Rappaport, "A geometrically based model for line-of-sight multipath radio channels," in Mobile Technology for the Human Race, vol. 2, pp. 844-848, IEEE, Atlanta, Ga, USA, April-May 1996, Proceedings of the 46th IEEE Vehicular Technology Conference.

[3] P. Petrus, J. H. Reed, and T. S. Rappaport, "Geometrical-based statistical macrocell channel model for mobile environments," IEEE Transactions on Communications, vol. 50, no. 3, pp. 495502, 2002.

[4] T. S. Rappaport, G. R. MacCartney, M. K. Samimi, and S. Sun, "Wideband millimeter-wave propagation measurements and channel models for future wireless communication system design," IEEE Transactions on Communications, vol. 63, no. 9, pp. 3029-3056, 2015.

[5] T. S. Rappaport, E. Ben-Dor, J. N. Murdock, and Y. Qiao, "Proceeding of the $38 \mathrm{GHz}$ and $60 \mathrm{GHz}$ angle-dependent propagation for cellular \& peer-to-peer wireless communications," in IEEE International Conference on Communications (ICC '12), pp. 4568-4573, Ottawa, ON, Canada, June 2012.

[6] T. S. Rappaport, F. Gutierrez, E. Ben-Dor, J. N. Murdock, Y. Qiao, and J. I. Tamir, "Broadband millimeter-wave propagation measurements and models using adaptive-beam antennas for outdoor Urban cellular communications," IEEE Transactions on Antennas and Propagation, vol. 61, no. 4, pp. 1850-1859, 2013.

[7] E. Ben-Dor, T. S. Rappaport, Y. Qiao, and S. J. Lauffenburger, "Millimeter-wave $60 \mathrm{GHz}$ outdoor and vehicle AOA propagation measurements using a broadband channel sounder," in
Proceedings of the 54th Annual IEEE Global Telecommunications Conference: "Energizing Global Communications" (GLOBECOM '11), Kathmandu, Nepal, December 2011.

[8] G. R. Maccartney Jr. and T. S. Rappaport, " $73 \mathrm{GHz}$ millimeter wave propagation measurements for outdoor urban mobile and backhaul communications in New York City," in Proceedings of the 1st IEEE International Conference on Communications (ICC '14), pp. 4862-4867, Sydney, Australia, June 2014.

[9] Z. Zhang, X. Chai, K. Long, A. V. Vasilakos, and L. Hanzo, "Full duplex techniques for 5G networks: self-interference cancellation, protocol design, and relay selection," IEEE Communications Magazine, vol. 53, no. 5, pp. 128-137, 2015.

[10] J. F. Schmidt, M. K. Atiq, U. Schilcher, and C. Bettstetter, "Underlay device-to-device communications in LTE-A: uplink or downlink?" in Proceedings of the 26th IEEE Annual International Symposium on Personal, Indoor, and Mobile Radio Communications (PIMRC '15), pp. 1542-1546, Hong Kong, China, September 2015.

[11] Y. Wang and J. P. Coon, "Outage probability of fixed-gain dual-hop relay selection channels with heterogeneous fading," Eurasip Journal on Wireless Communications and Networking, vol. 2015, article 195, 2015.

[12] O. Bulakci, Z. Ren, C. Zhou et al., "Towards flexible network deployment in 5G: Nomadic node enhancement to heterogeneous networks," in Proceedings of the IEEE International Conference on Communication Workshop (ICCW '15), pp. 25722577, London, UK, June 2015.

[13] R. He, A. F. Molisch, F. Tufvesson, Z. Zhong, B. Ai, and T. Zhang, "Vehicle-to-vehicle propagation models with large vehicle obstructions," IEEE Transactions on Intelligent Transportation Systems, vol. 15, no. 5, pp. 2237-2248, 2014.

[14] X. Yu, J. Zhang, and K. Letaief, "Coverage analysis for dense millimeter wave cellular networks: the impact of array size," in Proceedings of the Wireless Communications and Networking Conference (WCNC '16), vol. 3, pp. 1-6, Doha, Qatar, April 2016.

[15] S. Yoo, S. Cotton, R. Heath Jr., and Y. Chun, "Measurements of the $60 \mathrm{GHz}$ UE to eNB Channel for small cell deployments, IEEE Wireless Communications Letters," no. 99, pp. 1-4, 2017.

[16] D. Beauvarlet and K. L. Virga, "Measured characteristics of 30$\mathrm{GHz}$ indoor propagation channels with low-profile directional antennas," IEEE Antennas and Wireless Propagation Letters, vol. 1, pp. 87-90, 2002.

[17] J. Reig, M.-T. Martínez-Inglés, L. Rubio, V.-M. RodrigoPeñarrocha, and J. Molina-García-Pardo, "Fading evaluation in the $60 \mathrm{GHz}$ band in line-of-sight conditions," International Journal of Antennas and Propagation, vol. 2014, Article ID 984102, 12 pages, 2014.

[18] S. Panic, M. Stefanovic, J. Anastasov, and P. Spalevic, Fading and Interference Mitigation in Wireless Communications, CRC Press, Boca Raton, Fla, USA, 1st edition, 2013.

[19] E. Belding-Royer, A. Khaldoun, and G. Pujolle, Mobile and Wireless Communication Networks, Paris, France, 2005.

[20] J. A. Greenwood and D. Durand, "Aids for fitting the gamma distribution by maximum likelihood," Technometrics, vol. 2, no. 1, pp. 55-65, 1960.

[21] J. Cheng and N. C. Beaulieu, "Maximum-likelihood based estimation of the nakagami $\mathrm{m}$ parameter," IEEE Communications Letters, vol. 5, no. 3, pp. 101-103, 2001.

[22] A. Abdi and M. Kaveh, "Performance comparison of three different estimators for the nakagami $\mathrm{m}$ parameter using monte carlo simulation," IEEE Communications Letters, vol. 4, no. 4, pp. 119-121, 2000. 
[23] Y. Chen and N. Beaulieu, "Estimation of Ricean and Nakagami distribution parameters using noisy samples," in Procceeding of the IEEE International Conference on Communications (ICC '04), pp. 562-566, Paris, France, June 2004.

[24] Y. Chen, N. C. Beaulieu, and C. Tellambura, "Novel Nakagamim parameter estimator for noisy channel samples," IEEE Communications Letters, vol. 9, no. 5, pp. 417-419, 2005.

[25] N. Wang, X. Song, and J. Cheng, "Generalized method of moments estimation of the nakagami-m fading parameter," IEEE Transactions on Wireless Communications, vol. 11, no. 9, pp. 3316-3325, 2012.

[26] Y.-C. Ko and M.-S. Alouini, "Estimation of nakagami-m fading channel parameters with application to optimized transmitter diversity systems," IEEE Transactions on Wireless Communications, vol. 2, no. 2, pp. 250-259, 2003.

[27] I. S. Gradshteyn and I. M. Ryzhik, Table of Integrals, Series, and Products, Academic Press, 2007. 


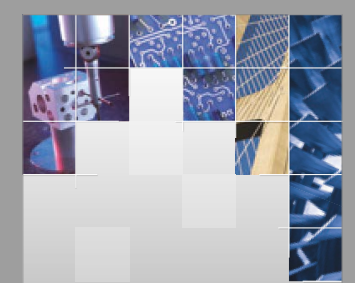

\section{Enfincering}
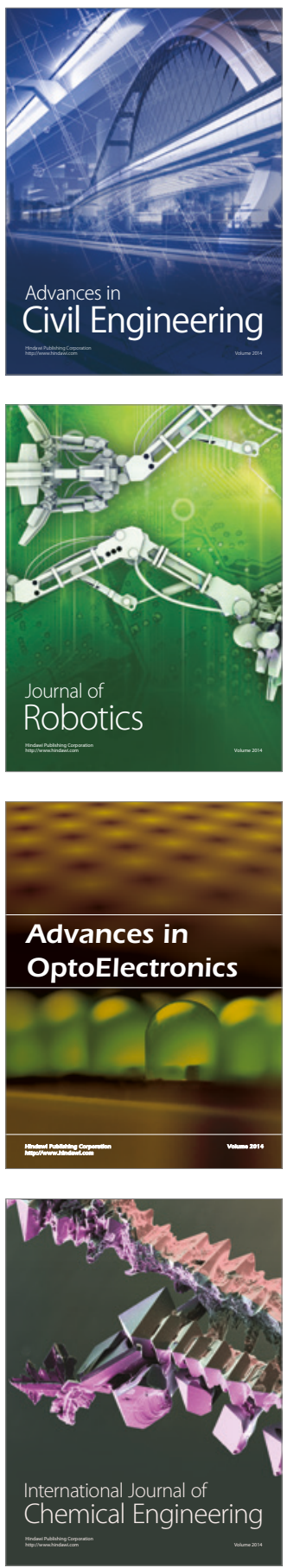

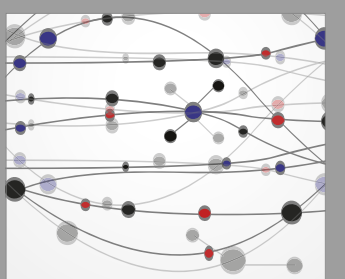

The Scientific World Journal

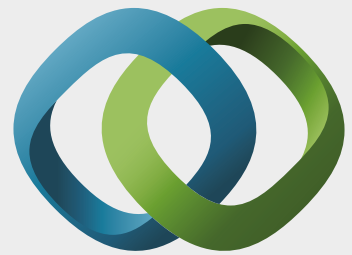

\section{Hindawi}

Submit your manuscripts at

https://www.hindawi.com
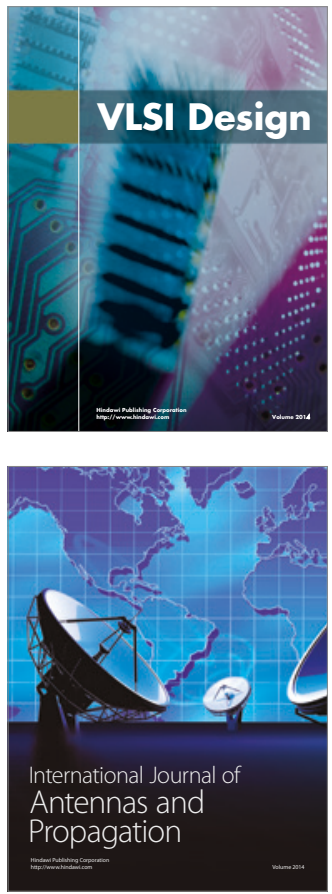

\section{Rotating}

Machinery
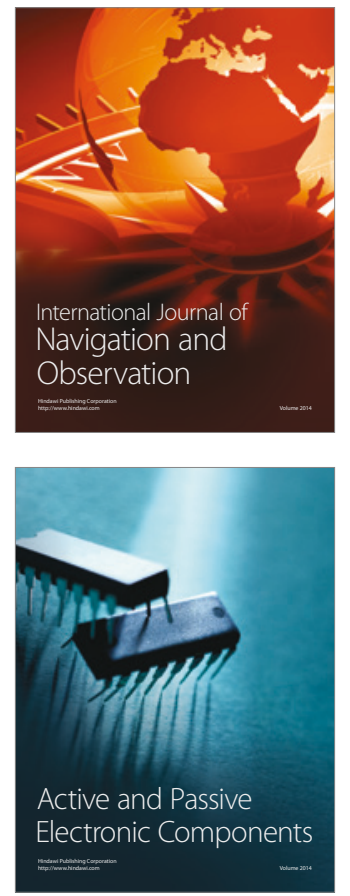
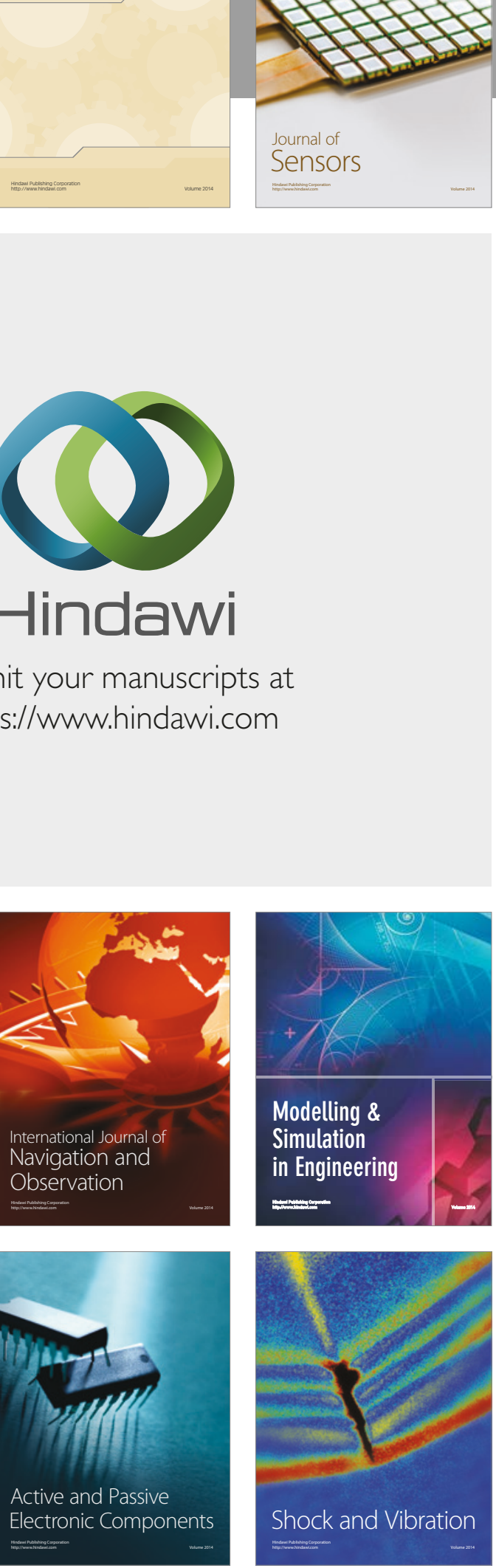
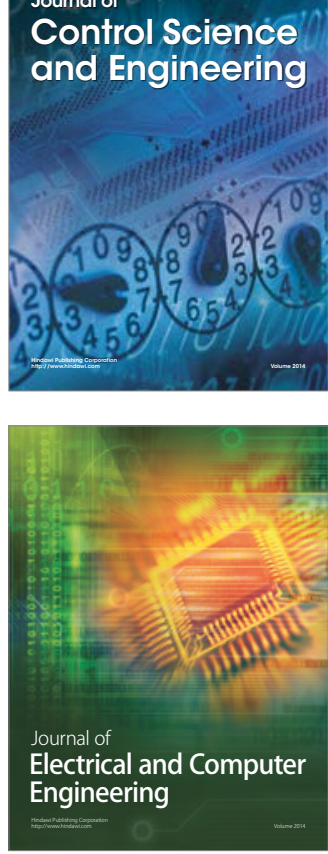

Distributed

Journal of

Control Science

and Engineering
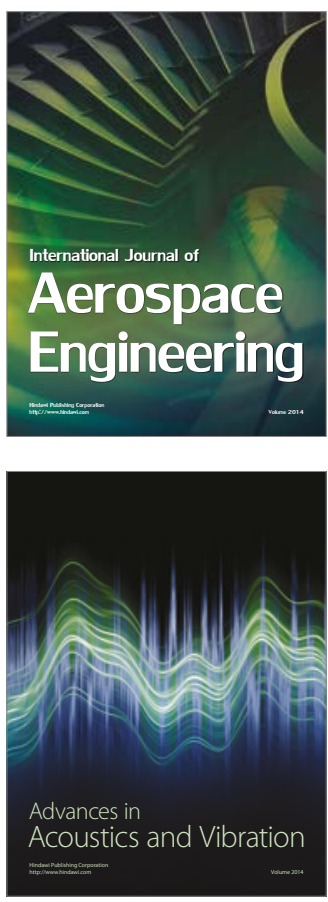

Sensor Networks 